\title{
Probing Fermionic Condensates by Fast-Sweep Projection onto Feshbach Molecules
}

\author{
S. Matyjaśkiewicz, ${ }^{1,2}$ M. H. Szymańska, ${ }^{3,4}$ and K. Góral ${ }^{5}$ \\ ${ }^{1}$ Department of Physics, King's College London, London WC2R 2LS, UK \\ ${ }^{2}$ Department of Applied Mathematics, Warsaw University of Life Sciences, Nowoursynowska 159, 02-776 Warsaw, Poland \\ ${ }^{3}$ Department of Physics, University of Warwick, Coventry CV4 7AL, UK \\ ${ }^{4}$ Cavendish Laboratory, University of Cambridge, Cambridge CB3 OHE, UK \\ ${ }^{5}$ Clarendon Laboratory, Department of Physics, University of Oxford, Parks Road, Oxford OX1 3PU, UK
}

(Dated: November 17, 2018)

\begin{abstract}
Fast sweep projection onto Feshbach molecules has been widely used as a probe of fermionic condensates. By determining the exact dynamics of a pair of atoms in time varying magnetic fields, we calculate the number of condensed and noncondensed molecules created after fast magnetic field sweeps from the BCS to the BEC side of the resonances in ${ }^{40} \mathrm{~K}$ and ${ }^{6} \mathrm{Li}$, for different sweep rates and a range of initial and final fields. We discuss the relation between the initial fermionic condensate fraction and the molecular condensate fraction measured after the sweep.

PACS numbers: 03.75.Ss, 03.75.Kk, 05.30.Fk
\end{abstract}

Ever since the first realisations of fermionic condensation in two-component gases of ${ }^{40} \mathrm{~K}\left[1\right.$ and ${ }^{6} \mathrm{Li}$ [2] the question how these fragile states can be accurately probed has been of great experimental importance. Since most techniques rely on the time-of-flight expansion, during which the Cooper pairs dissociate, indirect methods have been proposed. One of the most common is the so called fast-sweep projection [1, 3], which consists in a rapid magnetic field ramp from the BCS to the BEC side of the resonance. The key point is to make the field variation fast with respect to the many-body time scales thus preventing pair collisions from changing the pairs' momentum distribution and re-thermalisation. With this condition, the condensed molecules measured after the sweep originate only from the Cooper pairs, already present at the initial time, whereas the noncondensed molecules are converted only from the noncondensed atoms. The expectation is that this allows the information about the initial state to be easily unravelled from the measurements of molecules created during the sweep. The aim of this Letter is to describe the fast sweep regime of fermion dynamics and to determine whether and how the information about the initial condensate can be extracted from the production efficiency and the distribution of molecules after the sweep.

Despite its experimental relevance and fundamental interest, understanding of the dynamics of a cold Fermi gas following variations in the interaction strength is still relatively limited. The early analyses of molecular production were based on overlapping the initial pair state with the final molecular wave function [4]. This corresponds to an abrupt jump of the magnetic field strength and thus cannot account for the sweep rate dependence. The analysis of time-dependent processes has so far concentrated on the mean-field dynamics [5], which by definition ignores the noncondensed molecules. Thus, such methods are inadequate for sweeps where large numbers of noncondensed molecules were reported to be created during the ramp 3]. Finally, in [6] the dependence of the molecular production on the sweep rate was estimated analytically without determining the actual dynamics. This was done by overlapping the initial state with a sweep-ratedependent "final state" molecular wave function. Clearly, more rigorous calculations, which would determine the dynamics of the gas in time-varying fields and allow us to capture both condensed and noncondensed molecules created during this process, are still missing.

Making use of a systematic cumulant expansion [7], one can obtain the dynamic equations for the density matrix and the pair function of the gas. On this basis, the number of molecules beyond the mean-field level, i.e. accounting for noncondensed pairs, can be calculated. In this Letter we numerically solve these equations for a limited case of fast sweeps, during which pair collisions are insignificant. In such a case, the dynamical aspects are captured by the two-body time evolution of a single atom pair in the spirit of the original rationale behind the fast sweep projection technique. We determine the two-body time evolution exactly by numerically solving the time-dependent Schrödinger equation for a pair of atoms. We start from the single-channel Hamiltonian

$$
\hat{H}=\sum_{\mathbf{k} s} \epsilon_{\mathbf{k}} a_{\mathbf{k} s}^{\dagger} a_{\mathbf{k} s}+\sum_{\mathbf{k k}^{\prime} \mathbf{q}} V_{\mathbf{k k}^{\prime}} a_{\mathbf{k}+\mathbf{q} \uparrow}^{\dagger} a_{-\mathbf{k} \downarrow}^{\dagger} a_{-\mathbf{k}^{\prime} \downarrow} a_{\mathbf{k}^{\prime}+\mathbf{q} \uparrow},
$$

with Fermi operators $a_{\mathbf{k} s}^{\dagger}$ and $a_{\mathbf{k} s}$ (hereafter $\mathbf{k}, \mathbf{k}^{\prime}$, and $\mathbf{q}$ denote wave vectors in three dimensions). In the following we use a finite-range potential of the form $V_{\mathbf{k} \mathbf{k}^{\prime}}=V_{0}(B) \chi(\mathbf{k}) \chi\left(\mathbf{k}^{\prime}\right)$. Here $\chi(\mathbf{k})=\exp \left[-\left(\mathbf{k} \sigma_{\mathrm{bg}}\right)^{2} / 2\right]$ and the parameters $V_{0}(B)$ and $\sigma_{\mathrm{bg}}$ are chosen to recover the magnetic field dependence of the scattering length $a(B)=a_{\mathrm{bg}}\left(1-\frac{\Delta B}{B-B_{0}}\right)$ and of the highest vibrational bound state energy beyond the universal regime (details in $[8,9,[10])$. The density of molecules at time $t[11]$ is

$$
\begin{aligned}
n_{m}(\mathbf{q}, t)=\int d \mathbf{k} d \mathbf{k}^{\prime} \phi_{\mathrm{b}}^{\star}(B, \mathbf{k}) \phi_{\mathrm{b}}\left(B, \mathbf{k}^{\prime}\right) \\
\left\langle a_{\mathbf{k}+\mathbf{q} \uparrow}^{\dagger} a_{-\mathbf{k} \downarrow}^{\dagger} a_{-\mathbf{k}^{\prime} \downarrow} a_{\mathbf{k}^{\prime}+\mathbf{q} \uparrow}\right\rangle_{t},
\end{aligned}
$$


where $\phi_{\mathbf{b}}(B, \mathbf{k})$ is the Feshbach molecule wave function (determined by solving the stationary Schrödinger equation for a pair of atoms with the finite-range potential $\left.V_{\mathbf{k k}^{\prime}}\right)$ and the time dependence of the field is given by $B(t)=B_{0}+\dot{B}\left(t-t_{0}\right)$. To calculate (2) with the timedependent Hamiltonian (10) we use the cumulant expansion 7]. In the presence of a condensate, higher order correlation functions are still large, whereas higher order cumulants, which include the equivalent order of interactions around the interaction-free evolution, are small at least at short times. The expansion is thus controlled by how fast the resonance is crossed. Having expressed (2) in terms of cumulants, we can close the hierarchy of their dynamical equations by neglecting all fifth- and higher-order terms (the second-order expansion). Note, however, that since here $\left\langle a_{\mathbf{k s}}^{\dagger}\right\rangle=\left\langle a_{\text {ks }}\right\rangle=0$, we need to keep only the second-order cumulants (which here are equivalent to the correlation functions: the density ma$\operatorname{trix} \hat{\Gamma}(\mathbf{k}, t)=\frac{1}{2} \sum_{s}\left\langle a_{\mathbf{k s}}^{\dagger}(t) a_{\mathbf{k s}}(t)\right\rangle$ and the pair function $\left.\hat{\Phi}(\mathbf{k}, t)=\left\langle a_{-\mathbf{k} \downarrow}(t) a_{\mathbf{k} \uparrow}(t)\right\rangle\right)$ and the fourth-order ones of the form $\left\langle F_{1} F_{2} F_{3} F_{4}\right\rangle^{c}$ (where $F_{i}$ indicate any fermionic creation and annihilation operators). Next, by moving to the interaction picture, it is possible 7] to formally solve for all fourth-order cumulants and to obtain close equations for $\hat{\Gamma}(\mathbf{k}, t)$ and $\hat{\Phi}(\mathbf{k}, t)$. This results in additional non-Markovian collision terms containing products of $\hat{\Gamma}(\mathbf{k}, t)$ at different momenta and times in addition to terms already present in the mean-field equations [5]. Consequently, the molecular density becomes

$n_{m}(t)=\left.\left.\left|\int d \mathbf{k}\right|\left\langle\phi_{\mathrm{b}}(t) \mid \mathbf{k}\right\rangle\right|^{2} \hat{\Phi}(\mathbf{k}, t)\right|^{2}+$

$2\left\{\int d \mathbf{k}^{\prime}\left|T\left(\mathbf{k}^{\prime}, t, t_{0}\right)\right|^{2} \int d \mathbf{k} \hat{\Gamma}\left(\mathbf{k}, t_{0}\right) \hat{\Gamma}\left(\mathbf{k}-2 \mathbf{k}^{\prime}, t_{0}\right)+\right.$

$\left.\int_{t_{0}}^{t} d t^{\prime} \int d \mathbf{k}^{\prime}\left|T\left(\mathbf{k}^{\prime}, t, t^{\prime}\right)\right|^{2} \int d \mathbf{k} \frac{\partial}{\partial t^{\prime}}\left[\hat{\Gamma}\left(\mathbf{k}, t^{\prime}\right) \hat{\Gamma}\left(\mathbf{k}-2 \mathbf{k}^{\prime}, t^{\prime}\right)\right]\right\}$.

The two-body transition amplitude is defined as $T\left(\mathbf{k}, t, t_{0}\right)=\left\langle\phi_{\mathrm{b}}(t)\left|U_{2 \mathrm{~B}}\left(t, t_{0}\right)\right| \mathbf{k}\right\rangle$, where $U_{2 \mathrm{~B}}\left(t, t_{0}\right)$ is a two-body evolution operator. The first term in (3) describes condensed molecules and the remaining terms the noncondensed molecules created after time $t$. Secondorder cumulant expansion includes two-particle collisions but neglects higher order collision terms. Certainly, for very slow ramps, for which the re-thermalisation and multiple collisions are important, a higher order expansion may be needed. However, already the solution of full non-Markovian second order cumulant equations is numerically challenging and is beyond the scope of this Letter (although a suitable parallelisation of the computation should allow slower field variations to be studied). Here, our intention is to focus on the fast sweep limit. On such short time scales the evolution of the pair function $\hat{\Phi}$ is principally captured by the two-body evolution operator $U_{2 \mathrm{~B}}$, i.e $\hat{\Phi}(t)=U_{2 \mathrm{~B}}\left(t, t_{0}\right) \hat{\Phi}\left(t_{0}\right)$, and the third (collision) term in equation (3) is small. In this limit the density of condensed molecules becomes

$$
n_{m}^{c}(t)=\left|\int d \mathbf{k} T\left(\mathbf{k}, t, t_{0}\right) \hat{\Phi}\left(\mathbf{k}, t_{0}\right)\right|^{2},
$$

where $\hat{\Phi}\left(t_{0}\right)$ is the initial pair function. Density of noncondensed molecules is

$$
n_{m}^{n c}=2 \int d \mathbf{k}^{\prime}\left|T\left(\mathbf{k}^{\prime}, t, t_{0}\right)\right|^{2} \int d \mathbf{k} \hat{\Gamma}\left(\mathbf{k}, t_{0}\right) \hat{\Gamma}\left(\mathbf{k}-2 \mathbf{k}^{\prime}, t_{0}\right)
$$

where $\hat{\Gamma}\left(t_{0}\right)$ is the initial density matrix. We determine $U_{2 B}\left(t, t_{0}\right)$ exactly by numerically solving $i \hbar \frac{\partial}{\partial t} U_{2 B}\left(t, t_{0}\right)=\hat{H}_{2 B}(t) U_{2 B}\left(t, t_{0}\right)$, where $\hat{H}_{2 B}$ is equal to (11) for a pair of atoms. We are then in a position to evaluate $n_{m}^{c}$ and $n_{m}^{n c}$ from (4) and (5), respectively. Since our main focus is on establishing the relation between the final state after a fast sweep and the initial state, we take the simple mean-field thermodynamic initial conditions.

We first analyse the dependence of $n_{m}^{c}, n_{m}^{n c}$ and $n_{m}=$ $n_{m}^{c}+n_{m}^{n c}$ on the final field (Fig. 1). For finite-rate sweeps (unlike for the abrupt jump) the number of produced molecules depends on the final field only if it is in the region close to the resonance - it saturates further away from it; and the faster the sweep the further from the

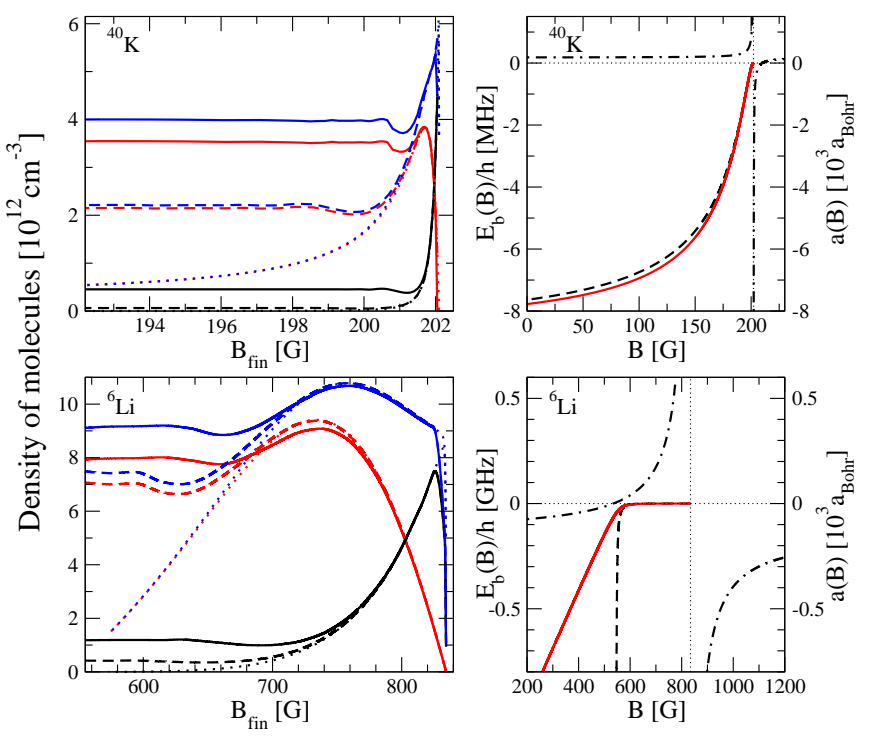

FIG. 1: (color online). Right: The scattering length (dasheddoted lines) and the bound state energy, $E_{\mathrm{b}}(B)$, of the ${ }^{40} \mathrm{~K}$ (top) and the ${ }^{6} \mathrm{Li}$ (bottom) Feshbach molecule $\phi_{\mathrm{b}}(B)$ in the vicinity of the $202 \mathrm{G}\left({ }^{40} \mathrm{~K}\right)$ and the $834 \mathrm{G}\left({ }^{6} \mathrm{Li}\right)$ resonances from two- (solid lines) and single-channel approaches (dashed lines). Left: Total (top curves, blue), condensed (middle curves, red) and noncondensed (bottom curves, black) molecular densities versus the final field, $B_{\text {fin }}$, for the initial field $B_{\text {ini }}-B_{0}=0.12 \mathrm{G}$ for ${ }^{40} \mathrm{~K}$ and $B_{\text {ini }}-B_{0}=4.62 \mathrm{G}$ for ${ }^{6} \mathrm{Li}$ and slew rates $(1 / \dot{B}): 10 \mu \mathrm{s} / \mathrm{G}\left({ }^{40} \mathrm{~K}\right), 0.01 \mu \mathrm{s} / \mathrm{G}\left({ }^{6} \mathrm{Li}\right)$ (solid lines); $1 \mu \mathrm{s} / \mathrm{G}\left({ }^{40} \mathrm{~K}\right), 0.003 \mu \mathrm{s} / \mathrm{G}\left({ }^{6} \mathrm{Li}\right)$ (dashed lines); and abrupt jump (dotted lines). The initial atomic densities are $1.5\left({ }^{40} \mathrm{~K}\right)$ and $2.9\left({ }^{6} \mathrm{Li}\right) 10^{13} \mathrm{~cm}^{-3}$. For ${ }^{40} \mathrm{~K}$ the parameters are from [1] and for ${ }^{6} \mathrm{Li}$ are motivated by [3, 12]. 
resonance this occurs. This supports the picture used in [6] that sufficiently far from the resonance $E_{b}$ is so large and interactions so small that the molecular state adiabatically follows the ramp (no molecules are created or dissociated during this part of the dynamics) 3]. For final fields near the resonance the molecular production is independent of the sweep rate and close to the one after jump. There is, however, an intermediate region of fields for which the molecular production is not yet saturated, but it depends on the rate. The presence of this region and also the exact position of the crossover to adiabatic dynamics is expected to be the main source of quantitative discrepancy between the dynamics presented here and analytical estimates from [6] for condensed pairs (additional differences arise for noncondensed ones - these are discussed later). Note, that there is a qualitative difference between the $202 \mathrm{G}$ resonance in ${ }^{40} \mathrm{~K}$ and the $834 \mathrm{G}$ resonance in ${ }^{6} \mathrm{Li}$ [10, 13] (see Fig 1). For ${ }^{6} \mathrm{Li}$ and slew rate $1 / \dot{B}=0.003 \mu \mathrm{s} / \mathrm{G}$ [17] used in [12] molecular production saturates around $250 \mathrm{G}$ below $B_{0}$, where the single and two-channel predictions already differ (there is around $15 \%$ difference in $E_{b}$ between the two). This suggests that if we were to study sweeps even faster than those in 12] an extension to two-channels would be necessary. In contrast, for ${ }^{40} \mathrm{~K} E_{b}$ from the single- and two-channel approaches coincide in the relevant region $B-B_{0}>-10 \mathrm{G}$ and are close for any field. Thus, the single channel theory is sufficient for analysis of molecular production in ${ }^{40} \mathrm{~K}$ for any sweep rate.
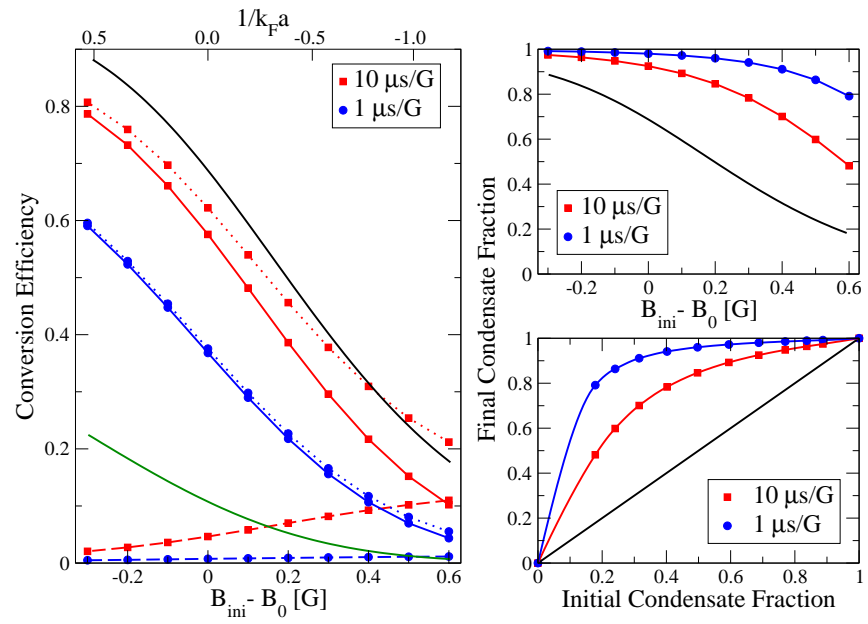

FIG. 2: (color online). Left: Molecular condensate, $2 n_{m}^{c} / n$, (solid lines), non-condensate, $2 n_{m}^{n c} / n$, (dashed lines) and total, $2 n_{m} / n$, (doted lines) conversion efficiencies versus the initial field, $B_{\text {ini }}$, for the final field $B_{\text {fin }}-B_{0}=-10 \mathrm{G}$ and two slew rates; and $2 n_{m}^{c} / n$ for an abrupt jump, which produces virtually no noncondensed molecules (bottom solid curve, green). Top solid curve (black) shows the initial (at $B_{\text {ini }}$ ) condensate fraction, $2 n_{c} / n$. Right: Final molecular condensate fraction, $n_{m}^{c} / n_{m}$, for the same parameters versus $B_{\text {ini }}$ (top), and versus the initial $2 n_{c} / n$ (bottom). Bottom solid curves (black) show the initial $2 n_{c} / n$. Calculations are for ${ }^{40} \mathrm{~K} 202 \mathrm{G}$ resonance and atomic density of $1.510^{13} \mathrm{~cm}^{-3}$.
The dependence of $n_{m}^{c}$ on the initial field (Fig. 2) is related to the number of Cooper pairs, $n_{c}(B)=$ $\int d \mathbf{k}|\hat{\Phi}(\mathbf{k}, B)|^{2}$, at that field, but it is different for different sweep rates. As expected, the number of condensed molecules, $n_{m}^{c}$, created during a linear sweep is always smaller than the number of initial Cooper pairs but larger than what one would obtain after an abrupt jump. The number of noncondensed molecules, $n_{m}^{n c}$, increases as the initial field is shifted deeper to the BCS side. This can be understood by noting that, in the fast sweep regime, the noncondensed pairs are created only from atoms which were initially out of the condensate and the number of these atoms increases towards the BCS side.

Finally, the dependence on the sweep rate is shown in Fig. 3 and compared with [6] and, for $n_{m}$, with the Landau-Zener (LZ) theory from [3]. Since Cooper pairs convert to molecules more efficiently than free atoms (as pointed out in [6]), LZ, which does not distinguish between the two, is unlikely to give an accurate account of molecular production from a partially condensed Fermi gas. Indeed, LZ results differ substantially from the results of our calculations (see Fig. 3). The differences with [6] are pronounced especially for the production efficiency of noncondensed molecules, which in [6] has a linear dependence on the slew rate with a coefficient dependent on the density but independent of the initial field. Here, it is instructive to compare our dynamics with an approach based on the asymptotic dissociation spectrum given by (B12) in [15]. It can be shown [15] that in the asymptotic limit of $t_{0} \rightarrow-\infty$ and $t \rightarrow+\infty$ the transition probability is given by $|T(\mathrm{k})|^{2}=\frac{\left|a_{\mathrm{bg}} \Delta B\right|}{\pi \hbar^{2} m|\dot{B}|} \exp \left(-\frac{4}{3} \frac{a_{\mathrm{bg}} \Delta B}{\hbar^{2} m \dot{B}} \mathrm{k}^{3}\right)$. Using this form instead of the exact one in (5) gives an approximate number of noncondensed molecules, good for $B_{\text {ini }}$ deeply in the BCS regime. An interesting case is that of $B_{\text {ini }}=B_{0}$ for which $n_{m}^{n c}$ obtained using the exact transition probability, $\left|T\left(\mathbf{k}, t, t_{0}\right)\right|^{2}$, turns out to be a half of $n_{m}^{n c}$ obtained using the asymptotic spectrum. On the basis of numerical evidence we can thus provide a simple formula for the number of noncondensed molecules for $B_{\text {ini }}=B_{0}$ as a function of the slew rate

$$
\begin{array}{r}
n_{m}^{n c} \approx \frac{\left|a_{\mathrm{bg}} \Delta B\right|}{2 \pi \hbar^{2} m|\dot{B}|} \int d \mathbf{k}^{\prime} \exp \left(-\frac{4}{3} \frac{a_{\mathrm{bg}} \Delta B}{\hbar^{2} m \dot{B}} \mathrm{k}^{\prime 3}\right) \\
\int d \mathbf{k} \hat{\Gamma}\left(\mathbf{k}, t_{0}\right) \hat{\Gamma}\left(\mathbf{k}-2 \mathbf{k}^{\prime}, t_{0}\right)
\end{array}
$$

(x symbols in Fig. 3), whose agreement with the exact one is very good indeed. The dependence on $1 /|\dot{B}|$ is a product of a linear term, dominant for very fast sweeps, and an exponential correction. The coefficient of this dependence is determined by the overlap of the transition probability and the initial density matrix at different momenta, and thus varies with the initial field (see Fig. 2).

As shown in Fig. 2 and 3 (Inset), the molecular condensate fraction, $n_{m}^{c} / n_{m}$, is always much larger than the initial one, $2 n_{c} / n$, but also it decreases to some extent 


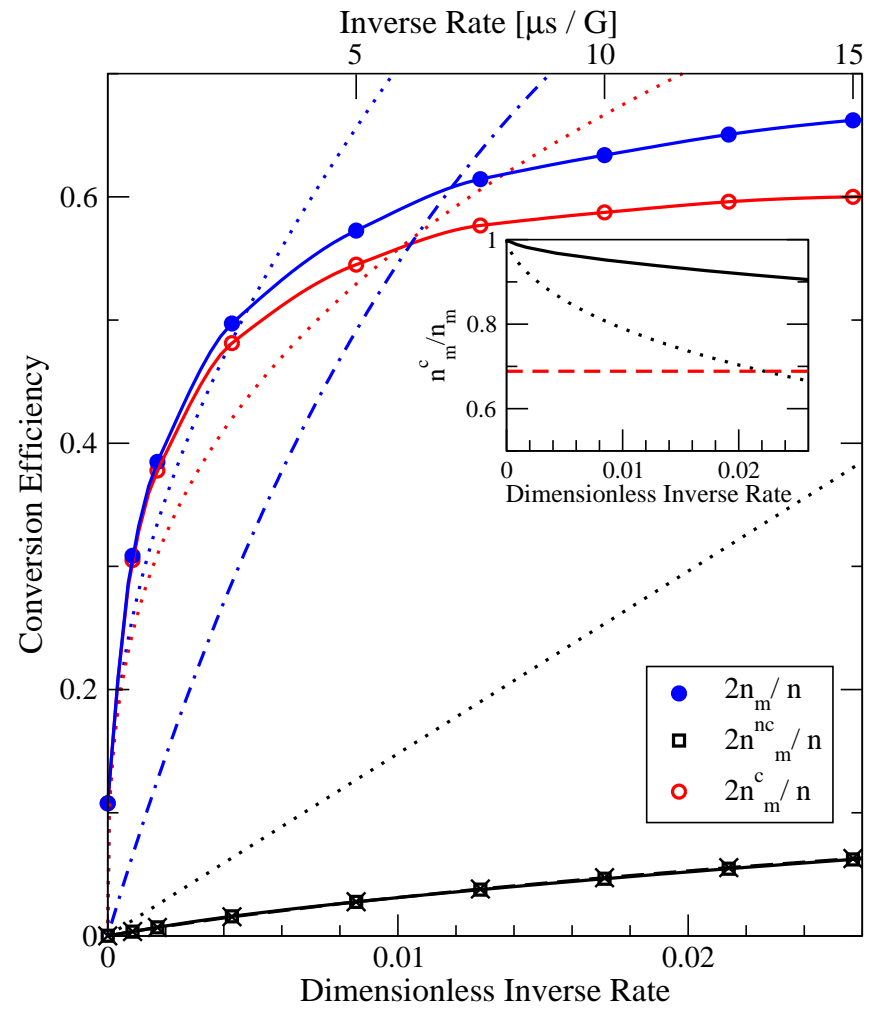

FIG. 3: (color online). Conversion efficiency for condensed, $2 n_{m}^{c} / n$, noncondensed, $2 n_{m}^{n c} / n$, and total, $2 n_{m} / n$, molecules versus the dimensionless inverse sweep rate $\frac{\hbar n \Delta B a_{b g}}{m} \frac{1}{B}$ for the ${ }^{40} \mathrm{~K} 202 \mathrm{G}$ resonance and atomic density of $1.510^{13} \mathrm{~cm}^{-3}$ for $B_{\text {ini }}=B_{0}$ and $B_{\text {fin }}-B_{0}=-10$ G. For comparison, formulas from [6] (dotted lines: total, condensed and noncondensed from top to bottom); and for $2 n_{m} / n$ the Landau-Zener prediction from [3] (dashed-dotted line) are shown. x symbols indicate the asymptotic approximation as discussed in the text. Inset: Final molecular condensate fraction, $n_{m}^{c} / n_{m}$, (solid line) for parameters as in the main Fig. For comparison, $n_{m}^{c} / n_{m}$ from [6] (dotted line) and the initial condensate fraction, $2 n^{c} / n$, (flat dashed line) are plotted.

with the slew rate. However, for slower sweeps the manybody effects become more important, which would again lead to an increase of the condensate fraction by formation of new condensed molecules from initially noncondensed atoms. This effect, together with three- and four-body collision losses which are density dependent and thus larger for condensed pairs occupying a smaller region in the centre of the trap [16], is a possible explanation for the sweep rate independent measurement of the condensate fraction in ${ }^{40} \mathrm{~K}$ (Fig. 5 in [1]). Note, that only the fastest sweeps in this Figure are expected to be in the fully two-body regime. Due to the large interchannel coupling it has proven to be much harder to realise the fast sweep limit for ${ }^{6} \mathrm{Li}$. For the fastest sweeps reported [12] of $0.003 \mu \mathrm{s} / \mathrm{G}$ (dashed lines in the left bottom panel of Fig. 1), atomic density $n=2.910^{13} \mathrm{~cm}^{-3}$ and initial field around $4 \mathrm{G}$ above the resonance, our method predicts around 0.49 for $2 n_{m}^{c} / n$ and about 0.03 for $2 n_{m}^{n c} / n$.

To conclude, we have shown that even in the limit of fast sweeps, where the dynamics of the gas is predominantly governed by the two-body evolution, the number of condensed molecules as well as the molecular condensate fraction after the sweep depend on both the sweep rate and the initial state. This makes it difficult to unravel the initial condensate fraction (and the initial number of Cooper pairs) from the measurement of the final molecular condensate without detailed dynamical calculations. However, although in the fast sweep limit the measured molecular condensate fraction always overestimates the initial condensate fraction, the presence of the molecular condensate implies the existence of the fermionic condensate before the sweep.

We are grateful to T. Köhler, K. Burnett, T. M. Hanna, A. F. Góral and M. W. Zwierlein for stimulating discussions. This research has been supported by Marie-Curie fellowship (S. M.), and EPSRC (M. H. S.).

[1] C. A. Regal, M. Greiner, and D. S. Jin, Phys. Rev. Lett. 92, 040403 (2004).

[2] M. W. Zwierlein et al., Phys. Rev. Lett. 92, 120403 (2004).

[3] for review see W. Ketterle and M. W. Zwierlein, arXiv:0801.2500 and references there in (2008).

[4] R. B. Diener, T. L. Ho, cond-mat/0404517 (2004), A. Perali, P. Pieri, and G. C. Strinati, Phys. Rev. Lett. 95, 010407 (2005).

[5] R. A. Barankov and L. S. Levitov, Phys. Rev. Lett. 93, 130403 (2004); R. A. Barankov, L. S. Levitov, and B. Z. Spivak, Phys. Rev. Lett. 93, 160401 (2004); A. V. Andreev, V. Gurarie, and L. Radzihovsky, Phys. Rev. Lett. 93, 130402 (2004); M. H. Szymańska, B. D. Simons, and K. Burnett, Phys. Rev. Lett. 94, 170402 (2005); E. A. Yuzbashyan, V. B. Kuznetsov, and B. L. Altshuler, Phys. Rev. B 72, 144524 (2005); E. A. Yuzbashyan, O. Tsyplyatyev, and B. L. Altshuler, Phys. Rev. Lett. 96, 097005 (2006); E. A. Yuzbashyan and M. Dzero, Phys. Rev. Lett. 96, 230404 (2006).

[6] E. Altman and A. Vishwanath, Phys. Rev. Lett. 95, 110404 (2005).

[7] T. Köhler and K. Burnett, Phys. Rev. A 65, 033601 (2002).

[8] K. Góral et al., J. Phys. B 37, 3457 (2004).

[9] T. Köhler, K. Góral, , and P. S. Julienne, Rev. Mod. Phys. 78, 1311 (2006).

[10] M. H. Szymańska et al. , Phys. Rev. A 72, 013610 (2005).

[11] T. Köhler, T. Gasenzer, and K. Burnett, Phys. Rev. A 67, 013601 (2003).

[12] L. Tarruell et al. , arXiv:cond-mat/0701181 (2005).

[13] W. Yi and L.-M. Duan, Phys. Rev. A 73, 063607 (2006).

[14] J. von Stecher and C. H. Greene, Phys. Rev. Lett. 99, 090402 (2007).

[15] T. M. Hanna et al., Phys. Rev. A 74, 023618 (2006).

[16] M. W. Zwierlein, Private communication (2007).

[17] Note that the relevant dimensionless slew rate which characterises the ramp speed is roughly $\frac{\hbar n \Delta B a_{b g}}{m} \frac{1}{B}$ [14] and thus $0.003 \mu \mathrm{s} / \mathrm{G}$ for ${ }^{6} \mathrm{Li}$ corresponds to around $12 \mu \mathrm{s} / \mathrm{G}$ for ${ }^{40} \mathrm{~K}$. 